\title{
Transfusional siderosis and liver cirrhosis
}

\author{
R. SINNIAH \\ From the Institute of Pathology, The Queen's University of Belfast
}

SYNOPSIS In the present study, cirrhosis of the liver was found in one of six cases of transfusional siderosis. The relationship between iron overload and cirrhosis is discussed and the suggestion is made that factors other than iron deposition may have been responsible for the hepatic fibrosis. Three patients with a greater degree of iron overload, and for a longer period, did not develop fibrosis or cirrhosis. The possibility remains that iron absorbed from the gut and stored in excess without having passed through the haemoglobin molecule or the reticuloendothelial system may be toxic, as cases have been reported in which cirrhosis of the liver was attributed to prolonged iron medication.

The cirrhosis in idiopathic haemochromatosis may be due to lack of an unknown hepatic cell enzyme, as in galactosaemia, and the increased iron absorption may be a concomitant finding. Transfusional or secondary haemochromatosis is not a definite entity, and the liver cirrhosis is probably due to causes other than the excess deposition of haemosiderin.

Since the association of excessive deposits of iron with cirrhosis of the liver and fibrosis of the pancreas was first described in haemochromatosis, it has been postulated that excessive accumulation of iron may be harmful to the tissues. The term 'siderosis' was coined by Neumann in 1888 , and originally it was applied to conditions in which stainable iron was found in the tissues, especially to conditions in which there was haemolysis. Sheldon (1935) emphasized the difference between haemosiderosis and haemochromatosis: haemosiderosis represented the deposition of iron pigment which was due to the destruction of red blood cells, and this was produced either experimentally or it resulted from a disease process. Finch and Finch (1955) defined haemochromatosis as iron deposition that caused cirrhosis and other tissue damage while in haemosiderosis the iron had not caused tissue damage. The pathological criteria used for the diagnosis of haemochromatosis were those proposed by MacDonald and Mallory (1960). They were as follows: (1) cirrhosis of the liver of a 'portal' type, with (2) excessive iron deposits in hepatic parenchymal cells, in connective tissue, and in bile duct epithelium; (3) pancreatic fibrosis and haemosiderosis; and (4) parenchymal iron deposits in other organs of the body.

The possibility that cirrhosis was due to iron pigment was first suggested by Hanot and Chauffard

Received for publication 31 December 1968 in 1882. They believed that iron and blood pigment acted as irritating substances which led to fibrosis. Sheldon (1935) also believed that iron set up an irritation which was followed by degeneration or atrophy of cells, the atrophied cells being replaced by fibrosis. But he considered that excessive destruction of blood and subsequent storage of iron would not cause haemochromatosis, but only a simple haemosiderosis without tissue damage. Thus, one theory is that cirrhosis is the result of the presence of iron pigment. Muir (1922) stated that cirrhosis of the liver and fibrosis of the pancreas occurred first, and this was followed by increased deposition of iron in the parenchymal cells of the organs and other damaged areas. A third theory is that cirrhosis and iron deposition are independent processes. In 1933, Polson after extensive studies in experimental animals in which he failed to produce tissue damage with iron, concluded that cirrhosis and iron pigmentation were independent.

This problem is investigated in the present study by assessment at necropsy of the effects of large quantities of iron retained in the liver and other tissues. These were cases of anaemias where life was sustained over long periods by multiple blood transfusions.

\section{MATERIALS AND METHODS}

Six cases of transfusional siderosis were selected from necropsies performed at the Royal Victoria Hospital and 
TABLE

ANALYSIS OF CASES OF TRANSFUSIONAL SIDEROSIS

\begin{tabular}{|c|c|c|c|c|c|c|c|c|}
\hline \multirow{2}{*}{ Case No. } & \multicolumn{2}{|c|}{ Sex and Age } & \multirow{2}{*}{ Blood Dyscrasia } & \multicolumn{2}{|c|}{ Amount Transfused } & \multirow[b]{2}{*}{$\begin{array}{l}\text { Duration of } \\
\text { Transfusion } \\
\text { (years) }\end{array}$} & \multirow[b]{2}{*}{$\begin{array}{l}\text { Cirrhosis } \\
\text { of } \\
\text { Liver }\end{array}$} & \multirow[b]{2}{*}{$\begin{array}{l}\text { Fibrosis } \\
\text { of } \\
\text { Pancreas }\end{array}$} \\
\hline & sex & ta Age & & $\begin{array}{l}\text { Blood } \\
\text { (pints) }\end{array}$ & $\begin{array}{l}\text { Iron } \\
(g)\end{array}$ & & & \\
\hline 1 & $\mathbf{F}$ & 46 & Refractory anaemia & 152 & $38 \cdot 0$ & 4 & 0 & 0 \\
\hline 2 & $\mathbf{M}$ & 73 & Chronic lymphocyt ic leukaemia & 32 & $8 \cdot 0$ & 4 & 0 & 0 \\
\hline 3 & $\mathbf{F}$ & 79 & Pancytopenia & 17 & $4 \cdot 3$ & $\overrightarrow{4}$ & 0 & 0 \\
\hline 4 & $\mathbf{M}$ & 72 & Sideroblastic anaemia & 23 & $17 \cdot 2^{1}$ & $3^{1}(8)$ & + & + \\
\hline 5 & $\mathbf{F}$ & 81 & Refractory anaemia & 116 & $29 \cdot 0$ & 10 & 0 & + \\
\hline 6 & $\mathrm{~F}$ & 78 & Sideroblastic anaemia & 96 & $24 \cdot 0$ & 5 & 0 & 0 \\
\hline
\end{tabular}

列 patient lived for eight years since start of blood transfusions, cirrhosis of the liver was found three years later.

City Hospital, Belfast. The clinical records were reviewed and all histological material was re-examined. The sections were stained with haematoxylin and eosin and Perls' Prussian blue reaction for haemosiderin in tissue. The details of the cases studied are summarized in the table, and the effect of iron on the liver and pancreas is also shown. In all six cases, multiple blood transfusions were given for blood dyscrasias: sideroblastic and refractory anaemias in four patients, pancytopenia and chronic lymphocytic leukaemia in the other two cases.

\section{RESULTS}

DISTRIBUTION OF IRON IN THE ORGANS The distribu-

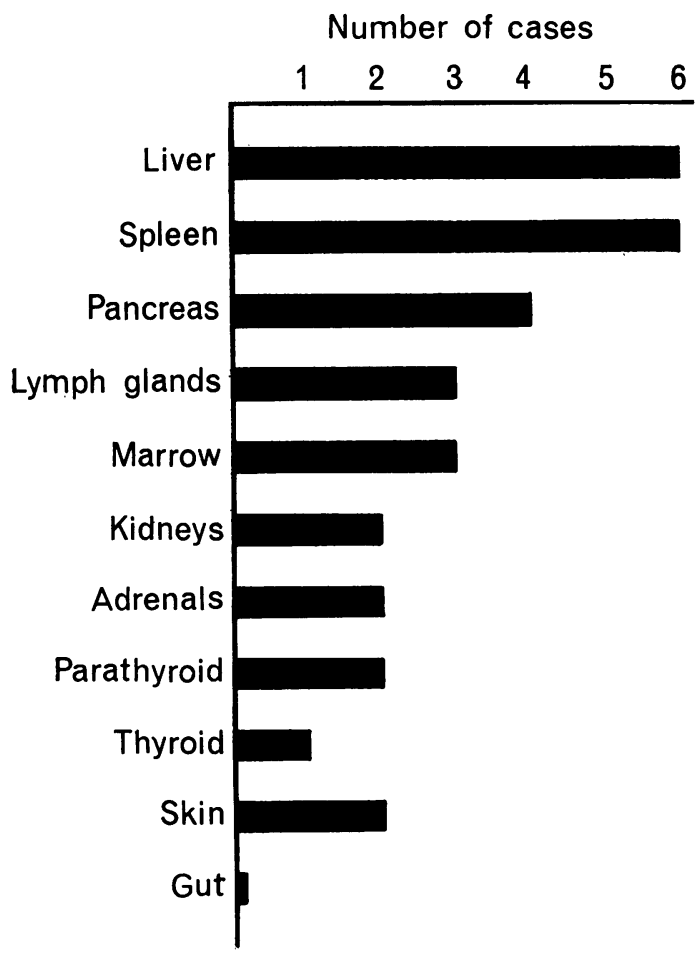

FIG. 1. Distribution of iron deposited in various organs in transfusional siderosis. tion of iron in the body in the six cases with trans- N fusional haemosiderosis is shown in Figure 1. The or maximum iron deposition was found in the liver and $\mathrm{v}$ spleen. The pancreas showed haemosiderin deposited in four of the cases. Only the two cases that had 음 received the largest amounts of blood had haemo- $\rightarrow$ siderin pigment in the dermis and around the skin $\mathscr{D}$ appendages. The distribution of iron in transfusional siderosis differed from that in idiopathic $\frac{\mathbb{D}}{3}$ haemochromatosis in that the maximum iron is found deposited in the reticuloendothelial system in the former condition, while in the latter haemosiderin $\vec{\theta}$ was deposited mainly in the parenchymal cells (Sinniah, 1967).

OCCURRENCE OF LIVER CIRRHOSIS From the study of $\overline{\bar{O}}$ the six cases of transfusional siderosis, liver cir- ڤ


pancreatic fibrosis was present in cases 4 and 5 . Case $\overrightarrow{\vec{A}}$ 4 closely resembled classical haemochromatosis at $\frac{0}{3}$ necropsy and was comparable to those cases previously recorded which have supported the belief that multiple blood transfusions may cause cirrhosis of the liver, with resultant 'secondary' or 'acquired' haemochromatosis. Before this case can be accepted as proof of hepatic cirrhosis caused by multiple blood transfusions, the case history and necropsy findings are more fully presented.

Clinico-pathological history of case 4 The patient $\frac{\text { Th }}{3}$ was a retired police officer, aged 72 years, who first $D$ presented in 1952 complaining of attacks of 'trembling' 을. for which no cause could be found. There was no clinical $\mathrm{N}$ evidence of anaemia at this time. $\mathrm{He}$ was seen again in $\Omega$ 1958 because of exertional dyspnoea, which had become $N$ increasingly severe during the previous eight months. On N examination he was found to be grossly anaemic and in $\omega$ congestive cardiac failure. The liver was enlarged to 1 in. $(2 \cdot 5 \mathrm{~cm})$ below the costal margin, but the spleen was not palpable.

The haemoglobin $(\mathrm{Hb})$ was $3.6 \mathrm{~g} / 100 \mathrm{ml}$, red cell count $\stackrel{\mathscr{S}}{+}$ $2,060,000 / \mathrm{cmm}$, PCV $16 \%$, MCHC $22 \%$ MCV $80 \mathrm{c} \mu$, reticulocytes $1 \%$. The white cells numbered $8,700 / \mathrm{c} \mathrm{mm}$, with a normal differential count. A sternal marrow aspirate showed a normoblastic hyperplasia with an $\frac{\Omega}{\mathbb{D}}$ orderly erythropoietic maturation and development. 
Liver function tests were normal. A fractional test meal revealed marked hyperchlorhydria. An $x$-ray barium meal examination suggested the presence of a small acute ulcer in the duodenum. As a consequence, the anaemia was attributed to gastrointestinal haemorrhage and treatment was directed towards correcting the anaemia by giving transfusions of concentrated red cells.

During the next four months, despite continuous iron therapy, it proved impossible to maintain the haemoglobin level, and repeated transfusions were necessary. In this period the patient received 23 pints of blood. Treatment with iron and ascorbic acid was recommenced, and the serum iron was found to be $470 \mu \mathrm{g} / 100 \mathrm{ml}$ and a total iron-binding capacity (TIBC) of $482 \mu \mathrm{g} / 100 \mathrm{ml}$. By this time his skin had acquired a slate-grey pigmentation, the liver had enlarged to 3 in. $(7 \cdot 5 \mathrm{~cm})$ below the costal margin, and the spleen had become palpable. His clinical condition had greatly deteriorated and was complicated by a return of congestive cardiac failure.

The patient had received at least the following amount of iron: 23 pints of blood $=5.75 \mathrm{~g}(500 \mathrm{ml}$ of blood contains approximately $250 \mathrm{mg}$ of iron), $130 \mathrm{ml}$ of Imferon $=7.50 \mathrm{~g}$, and $39 \mathrm{~g}$ of oral iron $=3.90 \mathrm{~g}$, on the assumption that he absorbed $10 \%$ of the oral dose (Chodos, Ross, Apt, Pollycove, and Halkett, 1957; PirzioBiroli, Bothwell, and Finch, 1958; Peterson, 1960). Thus the patient had received at least $17.20 \mathrm{~g}$ of iron. In addition to blood transfusions, $150 \mathrm{mg}$ of pyridoxine was given daily, with dramatic clinical improvement.
In 1961 a liver biopsy was performed, and it showed cirrhosis with marked haemosiderosis.

In 1964 there was still a good response to pyridoxine and the haemoglobin was satisfactory, but hepatomegaly and skin pigmentation were marked. The patient was given a short course of desferrioxamine-B to chelate iron from the liver as it was thought that the cirrhosis was due to the irritative action of haemosiderin. The patient became increasingly dyspnoeic and had some wheezy bronchitis with a lot of mucoid sputum, and he developed intermittent signs of right heart failure. Liver function tests were slightly abnormal and the serum iron level was $191 \mu \mathrm{g} / 100 \mathrm{ml}$. The liver was enlarged and extended to the umbilicus.

The patient died at home in 1966.

Necropsy findings (abnormal findings only) There was a generalized greyish-brown pigmentation of the skin, especially marked on the face, arms, and hands.

The liver was enlarged and weighed $2,000 \mathrm{~g}$. The surface was finely nodular, and on section the parenchyma had a rusty brown coloration, and there was a fine cirrhosis. On microscopic examination the lobular pattern was disrupted by coarse fibrous septa (Fig. 2) which subdivided the parenchyma into nodules of regenerating liver cells. The fibrous septa enclosed two or more lobules in some areas. Within the fibrous septa there was bile duct proliferation and a mild degree of haemosiderin deposition. In other areas, the fibrous septa were fine and surrounded single lobules. Haemosiderin was most

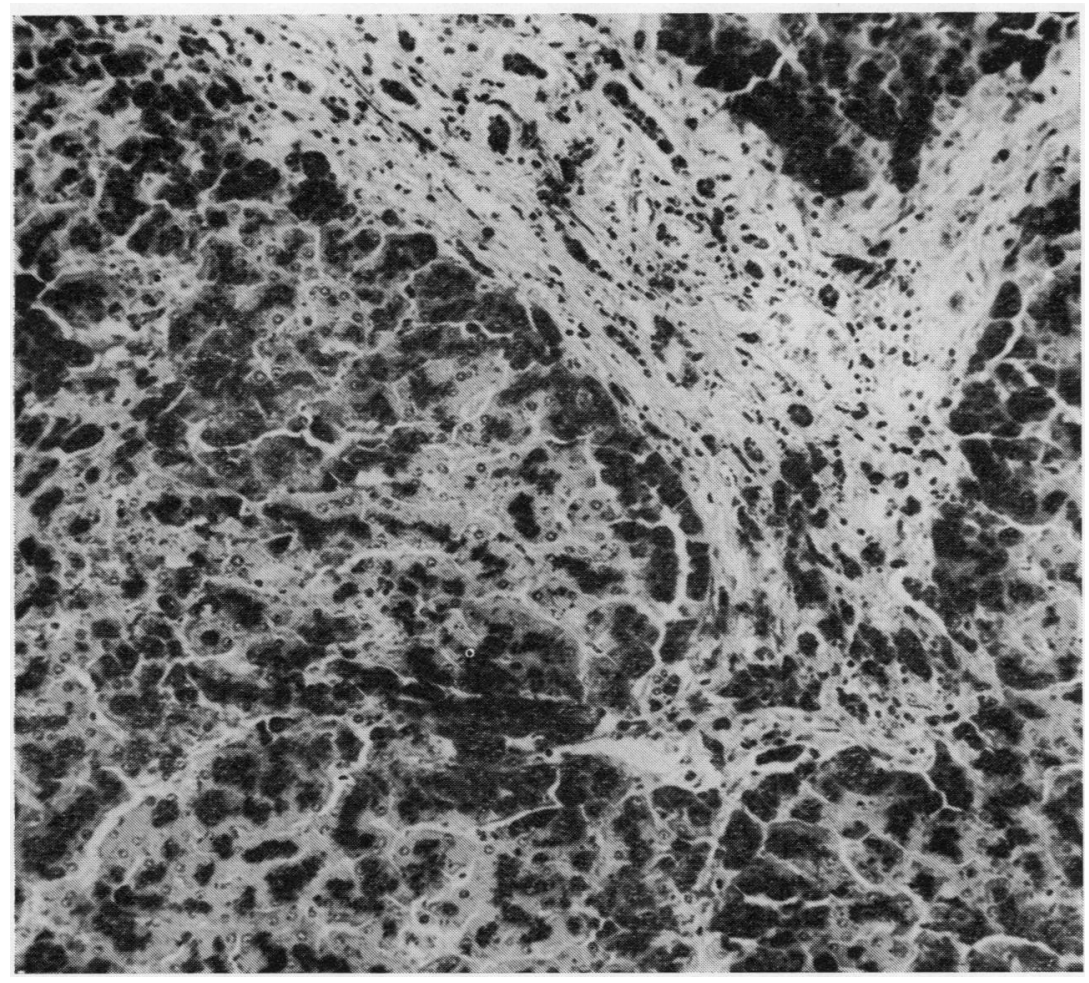

FIG. 2. Microscopic appearance of liver showing haemosiderosis and cirrhosis in a case treated by multiple blood transfusions (case 4). Perls' stain $\times 110$. 


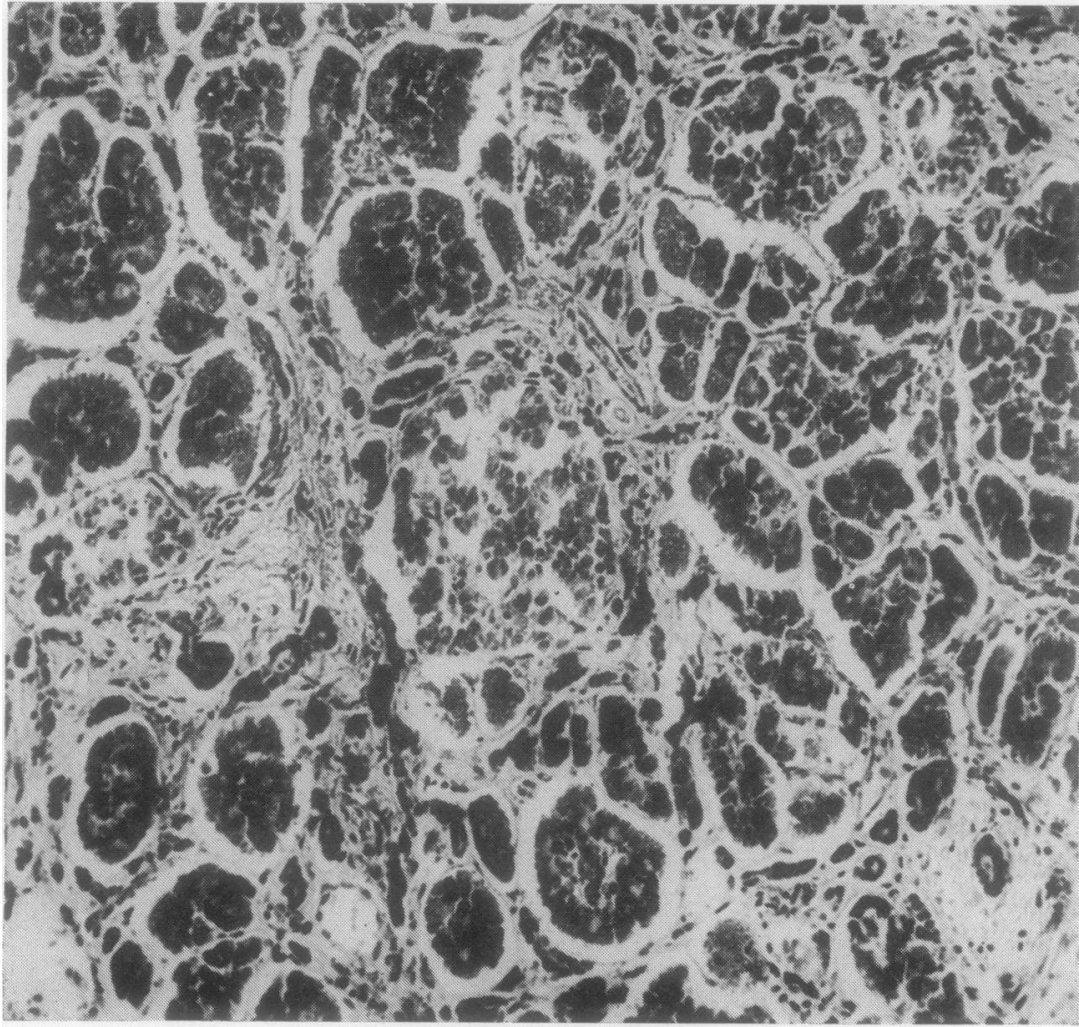

FIG. 3. Microscopic appearance of pancreas showing atrophy of acinar glands with interacinar fibrosis. Haemosiderin is present within the acinar cells and islets of Langerhan's (case 4). Perls' stain $\times 110$.

marked within the cytoplasm of the liver cells. The features were those of postnecrotic cirrhosis, though some areas were similar to portal cirrhosis and were consistent with a pathological diagnosis of 'haemochromatosis'.

The pancreas was a deep brown colour indicative of a heavy deposition of iron. The peripancreatic lymph nodes were also brown. On histological examination (Fig. 3) the architecture was seen to be disturbed due to a marked increase in interstitial fibrous tissue which ran between atrophic acini. Haemosiderin could be seen within the acinar cells and cells of the islets of Langerhans.

The spleen was moderately enlarged and weighed $400 \mathrm{~g}$. The pulp was congested and contained an excessive amount of haemosiderin.

Iron pigment was present in the cells of the zona glomerulosa of the adrenal glands.

Haemosiderin was deposited in an occasional tubule of the kidneys.

There was no deposition of haemosiderin in the myocardial fibres and no evidence of a myopathy was seen.

There was no excess melanin in the epidermis, and no iron was present in the skin appendages, dermis, and epidermis.

\section{DISCUSSION}

Hepatic cirrhosis with haemosiderosis was diagnosed $\overrightarrow{7}$ by a liver biopsy three years after the commence- ment of blood transfusions and both oral and 3 parenteral iron therapy. The patient had received at $\delta$ least $17 \cdot 2 \mathrm{~g}$ of iron into the tissues. Liver cirrhosis and pancreatic fibrosis were confirmed at necropsy five years later. The crux of the problem is to consider if hepatic cirrhosis and pancreatic fibrosis were caused by the haemosiderin acting as an irritant and inducing cellular damage or whether the $\sigma$ two conditions were separate.

The first case of haemochromatosis associated 요 with multiple blood transfusions was reported by $\sigma$ Kark in 1937. This occurred in a 39-year-old male who had received over 290 transfusions during a period of nine years. Since then, there have been a number of reports of so-called 'transfusion haemochromatosis' in patients who have received blood transfusions. Such patients have had anaemia- $\overrightarrow{\mathbb{D}}$ chiefly aplastic, haemolytic, and other anaemias. $\frac{\bigcirc}{\mathbb{D}}$ MacDonald (1964) cites a total of 128 patients in the 
literature with so-called transfusion haemochromatosis, but he states that only 13 cases have unequivocal cirrhosis of the liver, and the occurrence of only 13 cases does not suggest that cirrhosis is due to the blood and iron that was transfused, as the frequency is not greater than in many populations. MacDonald suggested that faulty nutrition, alcoholism, hepatitis as the result of transfusions, vascular congestion, and biliary obstruction were possible causes of cirrhosis, and had not been adequately considered in these cases.

Schwartz and Blumenthal (1948) reported five patients who died as a result of a variety of diseases, all characterized by severe anaemia for which numerous transfusions had been given, and all of whom developed features of haemochromatosis. They postulated that the haemochromatosis developing in these patients is the end result of the deposition and subsequent irritating action of iron in the cells. Cappell, Hutchison, and Jowett (1957), however, noted that Schwartz and Blumenthal had recorded as early periportal cirrhosis what was in fact the aggregation of macrophages laden with haemosiderin pigment. Finch, Hegsted, Kinney, Thomas, Rath, Haskins, Finch, and Fluharty (1950) reported the case of an 8-year-old male child with aplastic anaemia who had received 200 transfusions, containing $50 \mathrm{~g}$ of iron, in eight years. The child had liver cirrhosis. The authors concluded that iron in large amounts is toxic to tissues and causes fibrosis in organs most heavily laden with iron. Pengelly and Jones (1956) reported the case of a 20-year-old man who developed aplastic anaemia after exposure to benzol acetone and other solvents. This man received 360 bottles of blood, containing approximately $90 \mathrm{~g}$ of iron, over a nine-year period. He developed diabetes and a liver biopsy showed definite cirrhosis.

In the present study of six cases with transfusional siderosis, hepatic cirrhosis was found in only one patient (case 4). During a three-year period the patient received at least $17 \cdot 2 \mathrm{~g}$ of iron in the form of blood transfusions, oral and parenteral iron. Liver biopsy at the end of three years showed definite cirrhosis with severe haemosiderosis. Both hepatic cirrhosis and pancreatic fibrosis with haemosiderosis were confirmed at necropsy. Cases 1,5 , and 6 (see Table) had received at least $38 \mathrm{~g}, 29 \mathrm{~g}$, and $24 \mathrm{~g}$ of iron in blood transfusions and survived for periods of four, 10, and five years without the development of cirrhosis. Thus, if duration and intensity of iron therapy were the only factors concerned, the fibrosis should be most marked in those patients who had received the greatest amount of blood over the longest period, and this is not so.

Cappell et al (1957) reported five cases of trans- fusional siderosis. Two of their cases had received over 600 and 800 pints of blood, and had survived six and a half and 12 years respectively. Over $55 \mathrm{~g}$ of iron was present in the liver in each case, an amount twice that normally found in classical haemochromatosis (MacDonald, 1964), yet cirrhosis was absent. The authors remarked that iron derived from a large number of transfusions did not by itself reproduce the picture of haemochromatosis. Robbins (1958, quoted by MacDonald, 1964) reported a case of a 75-year-old female who had taken $2.7 \mathrm{~g}$ of ferrous sulphate daily for approximately 47 years. At necropsy, cirrhosis was present with iron in the liver and other organs. Turnberg (1965) published a case of a 60-year-old female who had anaemia 27 years previously, and had been on oral iron all the time, despite the disappearance of symptoms referable to anaemia. She developed hepatomegaly which extended to the umbilicus and a sallow complexion of the skin. A liver biopsy showed marked excess of iron pigment in parenchymal and Kupffer cells, and patchy fibrosis with bile duct proliferation. The total quantity of elemental iron consumed at a rate of $12 \mathrm{~g}$ daily for 27 years amounted to $12,000 \mathrm{~g}$.

From this study, and the findings of other workers, it would be incorrect to assume that siderosis alone, no matter how severe or long lasting, will of itself lead to cirrhosis or fibrosis and associated functional disturbances of true haemochromatosis. The liver cirrhosis and pancreatic fibrosis in the patient (case 4) investigated in this study may be due to factors other than the haemosiderin. Nine years before cirrhosis was detected the patient had attacks of 'trembling' for which no cause was found. Six years later, before blood transfusions and iron therapy were instituted, clinical examination revealed an enlarged liver. The initial symptom of 'trembling' may have been the rigor of infectious hepatitis, and hepatomegaly with liver cirrhosis may have already occurred when transfusions were started. At necropsy, microscopic examination of the liver revealed the features of postnecrotic cirrhosis. There was vascular congestion of the liver due to congestive cardiac failure. Thus, there was suggestive evidence that the liver cirrhosis was due to factors other than the presence of excess haemosiderin.

Finally, we have to consider what bearing these findings have on the difficult question of the relationship between iron deposition and fibrosis in classical haemochromatosis. In animal experiments, iron overload produced by a variety of methods has failed to induce fibrosis of organs (Cappell, 1930; Polson, 1933; Finch et al, 1950; Sinniah, 1967). In man with iron overload resulting from the treatment of non-regenerative anaemia by transfusion, there 
is a similar failure to demonstrate unequivocally a fibrogenic action of the transfused iron. According to Cappell et al (1957) it seems probable that at least some forms of iron are virtually non-fibrogenic. It may be possible that iron absorbed from the gut and stored in excess without having passed through the haemoglobin molecule may not be bland. The cases recorded by Robbins (1958) and Turnberg (1965), in which siderosis and hepatic cirrhosis were attributed to prolonged oral intake of iron for anaemia, may be cases in point, but these cases are still too few to arrive at a definite conclusion.

Thus, the behaviour in the body of excess iron derived from various sources may be different. Iron derived from the gut may be toxic and irritant when stored for long periods in excess, whereas iron derived from effete red cells may be relatively bland, having passed through the haemoglobin molecule (Cappell et al, 1957). Perhaps iron derived from the breakdown of red blood cells may not be irritant owing to its passage through the reticuloendothelial system. It may be possible that iron derived from any source is not toxic, and the cirrhosis in haemochromatosis is due to some other factor, possibly a specific but as yet unidentified hepatic enzyme comparable to what occurs in galactosaemia, in which the liver and red blood cells have been shown to lack the specific enzyme galactose-l-phosphateuridyl transferase which is essential for galactose metabolism (Kalckar, Anderson, and Isselbacher, 1956) and in which hepatic cirrhosis is found.
The author wishes to express his thanks to Professor Sir John H. Biggart, C.B.E., Professor Florence E. $\stackrel{\vec{F}}{\overrightarrow{0}}$ McKeown, and Mr D. W. Neill for their encouragementand help; to Dr M. G. Nelson for allowing me to study his cases; to $\mathrm{Mr} \mathrm{D}$. Mehaffey for the photographs; and to Miss M. Weller for secretarial assistance.

\section{REFERENCES}

Cappell, D. F. (1930). J. Path. Bact., 33, 175. Hutchison, H. E., and Jowett, H. (1957). Ibid, 74, 245.

Chodos, R. B., Ross, J. F., Apt, L., Pollycove, M., and Halkett, J. A. E. (1957). J. clin. Invest., 36, 314.

Finch, C. A., Hegsted, M., Kinney, T. D., Thomas, E. D., Rath 2 C. E., Haskins, D., Finch, S., and Fluharty, R. G.' (1950).त्] Blood, $5,983$.

Finch, S. C., and Finch, C. A. (1955). Medicine (Baltimore), 34, 381 N Hanot, V., and Chauffard, A. (1882). Rev. med. (Paris), 2, 385.

Kalckar, H. M., Anderson, E. P., and Isselbacher, K. J. (1956). Biochim. biophys. Acta (Amst.), $20,262$.

Kark, R. M. (1937). Guy's Hosp. Rep., 87, 343.

MacDonald, R. A., and Mallory, G. K. (1960), Arch. intern. Med $105,686$.

- (1964). Hemochromatosis and Hemosiderosis. C. C. Thomas, $\vec{\sim}$ Springfield, IIl.

Muir, R. (1922). Trans. roy. med.-chir. Soc. Glasg., 15, 107.

Neumann, E. (1888). Virchows Arch. path. Anat., 111, 25.

Pengelly, C. D. R., and Jones, P. (1956). Lancet, 2, 445.

Peterson, E. E. (1960). In The Metabolic Basis of Inherited Disease, edited by J. B. Stanbury, J. Wyngaarden and D. S. Fredrickson, p. 839. McGraw-Hill, Blakiston Div, New York.

Pirzio-Biroli, G., Bothwell, T. H., and Finch, C. A. (1958). J. Lab. $\vec{\bullet}$ clin. Med., 51, 37.

Polson, C. (1933). Brit. J. exp. Path., 14, 73.

Robbins, S. L. (1958). New Engl. J. Med., 258, 652. Quoted by MacDonald, R. A. (1964).

Schwartz, S. O., and Blumenthal, S. A. (1948). Blood, 3, 617.

Sheldon, J. H. (1935). Haemochromatosis. Oxford University Press, London.

Sinniah, R. (1967). Iron Metabolism and Idiopathic Haemochromatosis. M.D. Thesis, Trinity College, University of Dublin.

Turnberg, L. A. (1965). Brit. med. J., 1, 1360. 